\title{
Gas-Forming Pyogenic Liver Abscess in a Splenectomized Adult with Diabetes
}

\author{
Anupam Kotwal*, Kevin Abraham, Maria Garcia and Michael Thompson
}

Department of Medicine, University of Massachusetts Medical School, 55 Lake Avenue North, Room H6-531, Worcester, MA 01655, USA

\begin{abstract}
Pyogenic liver abscess (PLA) is uncommon in North America. A recent study has reported that persons with diabetes have increased risk of experiencing PLA and a higher 30-day post discharge mortality rate, compared with those without diabetes. Poor glycemic control has been linked to a higher likelihood of gas-forming PLA and metastatic infection due to Klebsiella pneumoniae. Here we present a patient with a history of total pancreatectomy and splenectomy for intraductal papillary mucinous neoplasm (IPMN) complicated by poorly controlled diabetes mellitus, who developed a gas-forming pyogenic liver abscess and was found to have bacteremia with extendedspectrum beta lactamase (ESBL) producing $K$. pneumoniae. During her hospital course, the abscess changed in appearance to multiloculated. Management was initially with broad spectrum empiric antibiotics, later changed to ertapenem along with abscess drainage. Patients with diabetes are particularly at risk of a gas-forming PLA and may have atypical presentation. Due to significantly increased mortality and morbidity from a gas-forming PLA, empiric antibiotic therapy should be started without waiting for culture results. Improved glycemic control is essential for management because sustained hyperglycemia has been linked to a poor outcome in gas-forming PLA.
\end{abstract}

Keywords: Gas-forming; Liver abscess; Diabetes; Splenectomy

\section{Introduction}

Pyogenic liver abscess (PLA) has been commonly reported in Asian countries but is a relatively uncommon clinical entity in North America, with an annual incidence of approximately 2.3 cases per 100,000 populations [1]. Gas-forming PLA accounts for a small percentage of PLA but has a high fatality rate in spite of aggressive management [2]. It is uncommon in western countries but is often reported in Taiwan [2]. There has been an increase in the isolation of Klebsiella pneumoniae (K. pneumoniae) as the causative organism of PLA [3]. K. pneumoniae is an encapsulated organism; hence there is a higher risk of infection and progression of disease in an individual who has undergone a splenectomy. Obstructive biliary disease, abdominal infections, previous surgical procedures, and immune-compromising medical conditions (including cancer and liver cirrhosis) have been reported as being risk factors for PLA [1]. A recent study has reported that persons with diabetes have a 3.6-fold increased risk of experiencing PLA and a higher 30-day post discharge mortality rate, compared with those who did not have diabetes [4]. Poor glycemic control has been linked to a higher likelihood of gas-forming PLA and metastatic infection due to K. pneumoniae [5,6]. Diabetes may cause gastroparesis and impaired neutrophil phagocytic function, and this can ultimately lead to hepatic bacterial seeding and abscess formation [7]. The average diabetes prevalence in patients with PLA has been reported to be approximately $11 \%-15 \%$ in the United States and Europe [3,4].

Studies have evaluated the correlation between PLA and visceral malignancies. A recent study has reported that the incidence of gastrointestinal cancer increases more than 4-fold among patients with PLA, with colorectal cancer being the highest, followed by cancers of the biliary tract, pancreas, and small intestine [8]. Even though $K$. pneumoniae is commonly isolated from a patient with PLA, most of the isolates of this organism causing PLA are highly susceptible to antibiotics [3]. It is very important to treat gas-forming PLA immediately, because bacteremia and septic shock frequently occur in these patients and the mortality rate is high [2].

Here we present a patient with a history of poorly controlled diabetes mellitus, intra-ductal papillary mucinous neoplasm (IPMN) and splenectomy, who developed a gas-forming pyogenic liver abscess and was found to have bacteremia with extended-spectrum beta lactamase (ESBL) producing K. pneumoniae.

\section{Case Description}

A 75-year-old Caucasian lady was admitted to the hospital for intermittent fevers of one week duration, associated with chills, rigors, fatigue and myalgia. She did not report abdominal pain, dysuria, rash, vomiting or diarrhea. Her past medical history was significant for mitral regurgitation, atrial fibrillation, osteoporosis and diverticulosis. She had undergone a total pancreatectomy splenectomy, cholecystectomy and duodenectomy for IPMN ten years prior to presentation. She developed diabetes mellitus as a consequence of the pancreatectomy and was on a continuous subcutaneous insulin infusion (CSII) pump, with the most recent hemoglobin $\mathrm{A} 1 \mathrm{C}(\mathrm{HbAlC})$ being $8.2 \%$. On presentation, she was noted to be febrile at $38.6^{\circ} \mathrm{C}$ and had sinus tachycardia, conjunctival icterus, jaundice and a grade 3/6 holosystolic murmur at the cardiac apex. She did not have ascites, abdominal tenderness, lymphadenopathy or hepatomegaly. Laboratory workup revealed a low hemoglobin of $11.7 \mathrm{~g} / \mathrm{dL}$ (reference range is 12-16) and elevated blood leukocyte count of 37,000 per $\mathrm{mm}^{3}$ (reference range is 4,30010,300 ) with $94 \%$ neutrophils. Liver function tests were significant for an elevated AST of $220 \mathrm{IU} / \mathrm{L}$ (reference range is 10-40), elevated ALT of $125 \mathrm{IU} / \mathrm{L}$ (reference range is 10-40), elevated total bilirubin of 17

*Corresponding author: Anupam Kotwal, MBBS, MD, Resident Physician Department of Medicine, University of Massachusetts Medical School, 55 Lake Avenue North, Room H6-531, Worcester, MA 01655, USA, Tel: 508-615-3723; Fax: 774-442-6781; E-mail: anupam.kotwal@umassmemorial.org

Received June 08, 2015; Accepted July 21, 2015; Published July 25, 2015

Citation: Kotwal A, Abraham K, Garcia M, Thompson M (2015) Gas-Forming Pyogenic Liver Abscess in a Splenectomized Adult with Diabetes. J Diabetes Metab 6: 583. doi:10.4172/2155-6156.1000583

Copyright: $\odot 2015$ Kotwal A, et al. This is an open-access article distributed under the terms of the Creative Commons Attribution License, which permits unrestricted use, distribution, and reproduction in any medium, provided the original author and source are credited. 
Citation: Kotwal A, Abraham K, Garcia M, Thompson M (2015) Gas-Forming Pyogenic Liver Abscess in a Splenectomized Adult with Diabetes. J Diabetes Metab 6: 583. doi:10.4172/2155-6156.1000583

Page 2 of 4

$\mathrm{mg} / \mathrm{dL}$ (reference range is $0.3-1.2$ ), elevated direct bilirubin of $5.6 \mathrm{mg} /$ $\mathrm{dL}$ (reference range is 0.0-0.4), elevated alkaline phosphatase (ALP) of $354 \mathrm{IU} / \mathrm{L}$ (reference range is $30-115$ ) and low albumin of $2.8 \mathrm{~g} / \mathrm{dL}$ (reference range is 3.5-4.8). The neutrophilic leukocytosis and hepatocellular pattern of jaundice in addition to the febrile state suggested an infectious hepatic process. Blood cultures were drawn and a CT scan of the abdomen was performed which revealed a fluid and gas containing $6 \times 6 \mathrm{~cm}$ collection in the right hepatic lobe (Figure 1). This was concerning for a PLA with a gas-forming organism, hence empiric therapy with intravenous piperacillin/tazobactam was initiated. Ultrasound guided percutaneous trans-hepatic drainage of the abscess was also performed after which a pigtail catheter was inserted. A transthoracic echocardiogram showed moderate mitral regurgitation but no vegetations. Initial blood cultures resulted positive for $K$. pneumoniae, Escherichia coli (E.coli) and Clostridium perfringens (C. perfringens) within 10 hours, following which the antibiotic regimen was changed to ampicillin/sulbactam based on the sensitivity of the three isolated organisms. Cultures of the drainage from the PLA grew Enterococcus fecalis and E.coli. Her leukocyte count slowly trended down to 13,400 per $\mathrm{mm}^{3}$, and liver enzymes and bilirubin normalized. Repeat blood cultures remained negative for five days. The patient was tested for viral hepatitis including HbS Ag and HCV RNA which were negative. She demonstrated immunity against hepatitis B infection. The patient experienced improvement in her symptoms and remained afebrile without any chills or rigors. Due to clinical and laboratory improvement over a period of two weeks, she was transitioned to oral amoxicillin/clavulanate and discharged from the hospital.

On the day after discharge she experienced sudden onset shortness of breath and presented to the emergency department. At this time she was found to be febrile and tachypneic with worsened leukocytosis and elevated alkaline phosphatase. Chest CT scan demonstrated multiple bilateral upper lobe segmental pulmonary emboli. Anticoagulation with unfractionated heparin via intravenous route was initiated, later transitioned to oral warfarin. Venous duplex scan revealed a chronic proximal right lower extremity deep vein thrombus. Repeat CT scan of the abdomen demonstrated a change in the appearance of the abscess to multi-loculated (Figure 2). This was further confirmed by a magnetic resonance cholangio-pancreatogram (MRCP) revealing a complicated abscess with a hemorrhagic component (Figure 3) along with mild intrahepatic biliary duct dilatation. A percutaneous biliary drain was placed, and piperacillin/tazobactam and clindamycin were initiated. Blood cultures this time resulted positive for ESBL producing $K$. pneumoniae, so the antibiotic regimen was changed to ertapenem. We checked tumor markers, of which CA 19-9, CA 125 and Carcinoembryonic antigen were mildly elevated, and Alphafetoprotein was normal. She also developed a right sided exudative pleural effusion (Figure 4) without features of empyema and with negative culture and cytology. Subsequently, she underwent a biopsy of the liver parenchyma surrounding the PLA which demonstrated histological features of inflammation but not malignancy. The abscess fluid was also negative for malignant cells. Over a period of 2 weeks, her laboratory abnormalities normalized, she remained afebrile, jaundice resolved and subsequent blood cultures remained negative. She was discharged from the hospital to a rehabilitation facility. During both her hospital admissions, insulin administration via the CSII pump was continued but the patient was noted to require a higher total daily dose of insulin than her usual. She received intravenous ertapenem on an ambulatory basis for a total of 6 weeks. The pitgtail catheter and biliary drain were removed 3 weeks after discharge. The patient remained afebrile during clinic follow up and reported symptomatic improvement. Her laboratory abnormalities also normalized and the HbA1C improved to $7.1 \%$. On CT imaging, there was evidence of near complete resolution of the abscess, with only a $2 \mathrm{~cm} \times 1 \mathrm{~cm}$ lucency still remaining (Figure 5).

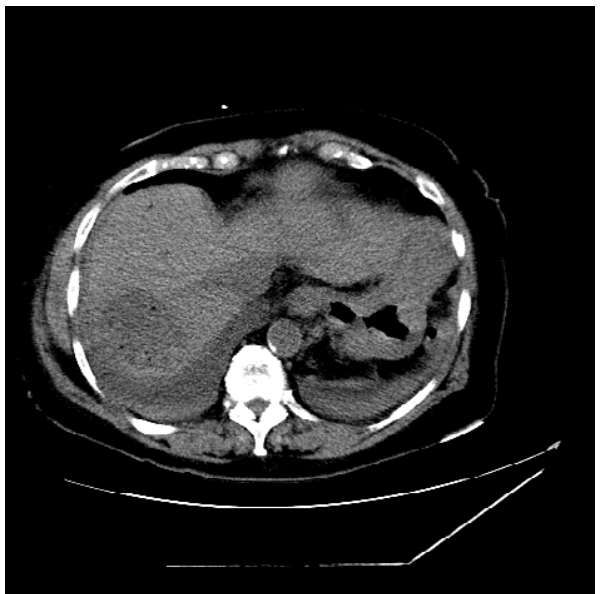

Figure 1: CT of the abdomen demonstrating a fluid and gas containing $6 \times 6$ $\mathrm{cm}$ hypodense lesion in the right hepatic lobe.

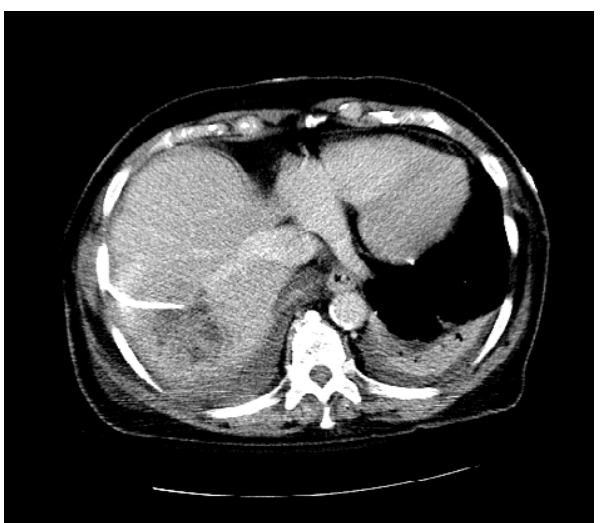

Figure 2: CT of the abdomen demonstrating a fluid and gas containing multiloculated $6 \times 6 \mathrm{~cm}$ hypodense lesion in the right hepatic lobe.

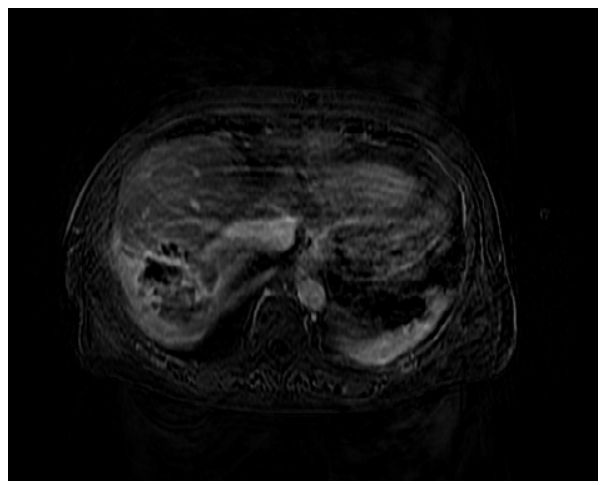

Figure 3: MRCP demonstrating a $6 \times 6 \mathrm{~cm}$ multiloculated lesion in the right hepatic lobe, with low signal on T1 and heterogenous signal on T2. 


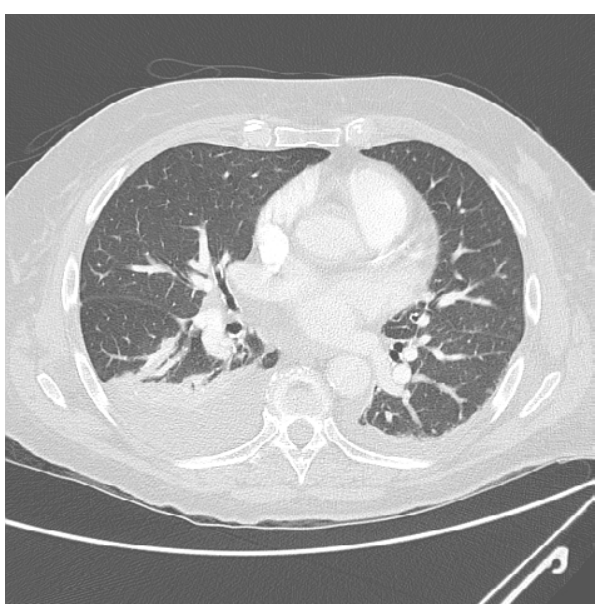

Figure 4: CT of the chest demonstrating right more than left sided pleural effusion.

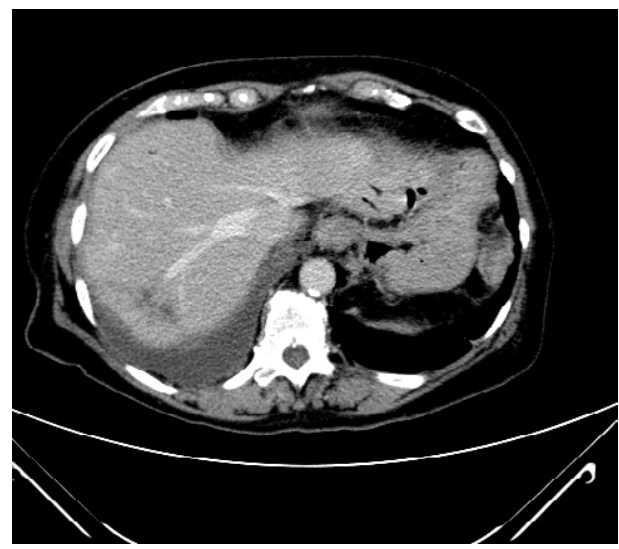

Figure 5: CT of the abdomen demonstrating near complete resolution of the abscess with only a $2 \mathrm{~cm} \times 1 \mathrm{~cm}$ lucency still remaining.

\section{Discussion}

Our patient did not present with the typical triad of clinical features historically characteristic of PLA, in that she did not demonstrate abdominal pain or tenderness. However, the prolonged febrile state along with neutrophilic leukocytosis and hepato-cellular pattern of jaundice suggested an infectious hepatic process. Our patient's history of poorly controlled diabetes, IPMN and abdominal surgery increased the risk of developing a gas-forming PLA. The absence of spleen increased her susceptibility to encapsulated organisms like K. pneumoniae. Poor glycemic control has been linked to a higher likelihood of gas-forming PLA and metastatic infection due to K. pneumoniae [5,6]. Diabetes mellitus is thought to promote colonic bacterial overgrowth secondary to gastroparesis and impaired cellular phagocytic dysfunction in $\mathrm{K} 1$ or K2 capsular serotype of K. pneumoniae infection [9]. Poor glycemic control is associated with impaired neutrophil bactericidal function [10]. The absence of abdominal pain or tenderness and the different organisms growing from the PLA versus the blood were unusual features in this case.

The CT scan and MRCP demonstrated features concerning for a gas-forming or emphysematous PLA. In patients with diabetes, the high level of blood glucose may provide gas-forming microorganisms with a more favorable environment for forming gas via mixed acid fermentation of glucose [11]. The major causative organisms responsible for gas-forming PLA in diabetic patients are K. pneumoniae [11] and E. coli, with rare cases due to C. perfringens. Although C. perfringens is relatively rare, the survival rate is very poor compared with those of K. pneumoniae and E. coli. Therefore, for every case that presents with a gas-forming PLA, the possibility of $C$. perfringens should be considered, and immediate aspiration of the abscess and Gram staining are important. The management of gas-forming PLA incorporates blood cultures, antibiotic therapy and abscess drainage. Empiric broad-spectrum parenteral antibiotics should be administered pending abscess gram stain and culture results, and should later be revised based on the isolated organisms. After drawing blood cultures from our patient, empiric therapy with piperacillin/tazobactam and clindamycin was initiated to cover for Gram negative organisms, anaerobes and the toxin produced by $C$. perfringens. It was surprising that the cultures from the abscess drainage did not grow K. pneumoniae or C. pefringens, however this was probably due to the initiation of antibiotics prior to abscess drainage.

There are no randomized controlled trials evaluating the optimal duration of antibiotic therapy for PLA. This is typically determined by the extent of infection and the patient's clinical response to initial management. Due to clinical and laboratory improvement after 2 weeks of intravenous antibiotics, our patient was transitioned to oral amoxicillin/clavulanate and discharged from the hospital, but soon readmitted with sepsis and pulmonary emboli. The isolation of ESBL producing $K$. pneumoniae from blood drawn while on oral antibiotics necessitated therapy with a carbapenem for a planned duration of 4-6 weeks. In addition to antibiotic therapy, drainage of the abscess is essential, which can be percutaneous, via fine needle aspiration, or surgical [12]. Percutaneous drainage and fine needle aspiration have been effective in the drainage of both single and multiloculated abscesses, and surgical drainage is performed when other techniques have failed [12]. The patient discussed here initially presented with a single gas-forming PLA that was drained with a percutaneous pigtail catheter in place. On readmission, subsequent imaging revealed a multiloculated abscess. On follow up over a period of 2 months, the patient demonstrated improvement in her symptoms, glycemic control and jaundice. She completed a 6 week course of intravenous ertapenem, remained afebrile and demonstrated near total resolution of the gas-forming PLA.

PLA has been shown to be associated with an increased incidence in visceral malignancies, most commonly colorectal carcinoma [8]. It is unclear whether our patient's history of being diagnosed with IPMN had any association with the development of the PLA. During her hospital course, mild elevation of tumor marker CA 199, CA 125 and Carcinoembryonic antigen was noted. However, the absence of histological evidence of malignancy from the liver and a recent normal colonoscopy suggest that this elevation was most likely due to an inflammatory state caused by the PLA. Our patient also developed an exudative right sided pleural effusion without features of malignancy, and bilateral pulmonary emboli. The pleural effusion could be explained by the inflammation of the adjacent tissue caused by the PLA. As far as the pulmonary emboli are concerned, the patient was found to have a chronic proximal right lower extremity deep vein thrombus which could have caused the emboli. However, it should be noted that the emboli could have been a complication of the gasforming PLA. It is very much possible that septic emboli from the PLA migrated to the lungs. This finding is supported by the recurrence of fever and the isolation of ESBL producing K. pneumoniae from repeat 
Citation: Kotwal A, Abraham K, Garcia M, Thompson M (2015) Gas-Forming Pyogenic Liver Abscess in a Splenectomized Adult with Diabetes. J Diabetes Metab 6: 583. doi:10.4172/2155-6156.1000583

Page 4 of 4

blood cultures that occurred concurrently with the development of the pulmonary emboli

\section{Conclusions}

Gas-forming PLA is an uncommon clinical entity in North America. K. pneumoniae, E. coli and C. perfringens have been implicated to be the causative organisms. The absence of abdominal pain or tenderness and the different organisms isolated from the abscess drainage versus blood were unusual features in this case. Patients with poorly controlled diabetes have an increased risk of developing a gas-forming PLA caused by K. pneumonia and may have an atypical presentation. Due to the significantly increased mortality and morbidity from a gas-forming PLA, it is imperative to initiate immediate empiric broad spectrum antibiotic therapy without waiting for culture results from the blood and abscess fluid. The duration of antibiotic therapy is typically determined by the extent of the infection and the patient's clinical response to initial management. Percutaneous drainage has been shown to be effective for single as well as multi-loculated abscesses, with surgical drainage being used as a last resort. The association of PLA with visceral malignancies and the risk of metastatic infection should be kept in mind during management and follow up. In addition to antibiotics and drainage of the abscess, improving glycemic control is an essential aspect of management of PLA.

\section{References}

1. Kaplan GG, Gregson DB, Laupland KB (2004) Population-based study of the epidemiology of and the risk factors for pyogenic liver abscess. Clin Gastroenterol Hepatol 2: 1032-1038.

2. Yang CC, Chen CY, Lin XZ, Chang TT, Shin JS, et al. (1993) Pyogenic live abscess in Taiwan: emphasis on gas-forming liver abscess in diabetics. Am J Gastroenterol 88: 1911-1915.
3. Rahimian J, Wilson T, Oram V, Holzman RS (2004) Pyogenic liver abscess: recent trends in etiology and mortality. Clin Infect Dis 39: 1654-1659.

4. Thomsen RW, Jepsen P, Sørensen HT (2007) Diabetes mellitus and pyogenic liver abscess: risk and prognosis. Clin Infect Dis 44: 1194-1201.

5. Lin YT, Wang FD, Wu PF, Fung CP (2013) Klebsiella pneumoniae liver abscess in diabetic patients: association of glycemic control with the clinical characteristics. BMC Infect Dis 13: 56.

6. Foo NP, Chen KT, Lin HJ, Guo HR (2010) Characteristics of pyogenic liver abscess patients with and without diabetes mellitus. Am J Gastroenterol 105: 328-335.

7. Ngo JT, Parkins MD, Gregson DB, Pitout JD, Ross T, et al. (2013) Populationbased assessment of the incidence, risk factors, and outcomes of anaerobic bloodstream infections. Infection 41: 41-48.

8. Lai HC, Lin CC, Cheng KS, Kao JT, Chou JW, et al. (2014) Increased incidence of gastrointestinal cancers among patients with pyogenic liver abscess: a population-based cohort study. Gastroenterology 146: 129-137.

9. Lin JC, Siu LK, Fung CP, Tsou HH, Wang JJ, et al. (2006) Impaired phagocytosis of capsular serotypes K1 or K2 Klebsiella pneumoniae in type 2 diabetes mellitus patients with poor glycemic control. J Clin Endocrinol Metab 91: 3084-3087

10. Gallacher SJ, Thomson G, Fraser WD, Fisher BM, Gemmell CG, et al. (1995) Neutrophil bactericidal function in diabetes mellitus: evidence for association with blood glucose control. Diabet Med 12: 916-920.

11. Lee HL, Lee HC, Guo HR, Ko WC, Chen KW (2004) Clinical significance and mechanism of gas formation of pyogenic liver abscess due to Klebsiella pneumoniae. J Clin Microbiol 42: 2783-2785

12. Liu CH, Gervais DA, Hahn PF, Arellano RS, Uppot RN, et al. (2009) Percutaneous hepatic abscess drainage: do multiple abscesses or multiloculated abscesses preclude drainage or affect outcome? J Vasc Interv Radiol 20: 1059-1065. 